\title{
Effects of a Single-Dose of Mind-Body Practice on the Heart Rate Variability, Brain Activity and Shooting Performance in Archers: A Pilot Study
}

\author{
Peter X. L. Li' ${ }^{1}$ Chung S. C. Chan², Kenneth K. Y. Lai' ${ }^{2}$, Jennifer P. C. Wong², William W. N. Tsang2 ${ }^{*}$ (1) \\ ${ }^{1}$ Medical School, Yangtze University, Jingzhou, China \\ ${ }^{2}$ Department of Rehabilitation Sciences, The Hong Kong Polytechnic University, Hong Kong, China \\ Email: *William.Tsang@polyu.edu.hk
}

How to cite this paper: Li, P.X.L., Chan, C.S.C., Lai, K.K.Y., Wong, J.P.C. and Tsang, W.W.N. (2019) Effects of a Single-Dose of Mind-Body Practice on the Heart Rate Variability, Brain Activity and Shooting Performance in Archers: A Pilot Study. Yangtze Medicine, 3, 124-134.

https://doi.org/10.4236/ym.2019.32012

Received: October 19, 2018

Accepted: March 19, 2019

Published: March 22, 2019

Copyright $\odot 2019$ by author(s) and Scientific Research Publishing Inc. This work is licensed under the Creative Commons Attribution International License (CC BY 4.0).

http://creativecommons.org/licenses/by/4.0/

(c) (i) Open Access

\begin{abstract}
Objective: Factors affecting performance of archery are categorized into psychological, skill, and fitness perspectives. Increase in attention, relaxation and parasympathetic system activity is believed to enhance the shooting performance of archers during competition. This pilot study aims to investigate the effects of single-dose of Tai Chi practice on archer's performance and the feasibility of an investigation protocol under a competition scenario. Methods: This was a randomized controlled trial (RCT) with a crossover study design. Pre- and post-intervention measurements were conducted to evaluate the effects of Tai Chi practice on the archer's autonomic nervous system activity, attention and relaxation through electroencephalography investigation, and shooting performance when compared with stretching as control. Results: The protocol of the study was found to be feasible during a competition setting. No significant difference was detected in the outcome measures under the two intervention conditions. Participants after practicing Tai Chi were found to improve significantly in their shooting performance $(\mathrm{p}=$ 0.021). Conclusion: This pilot study showed that a real-time measurement of autonomic nervous system and brain wave activities in the competition field was feasible and it did not hinder the archers' performance. There was a significant improvement observed in the shooting performance of archers after Tai Chi practice when compared with baseline. The findings of this pilot study provide the basis for the conduction of a larger RCT study to examine the potential beneficial effects of Tai Chi practice on archers.
\end{abstract}

\section{Keywords}

Mind-Body, Autonomic Nervous System, Brain Waves, Archery 


\section{Introduction}

Factors affecting performance of archery can be categorized into psychological, skill, and fitness perspectives [1]. Among them, archery experts believe that the psychological condition is the most important component in a match. Archery athletes with more than five years of experience have been regarded to possess similar fitness and skill.

According to the findings by Lee (2009) [2] using the Neurosky EEG headband system, elite archers had attained a certain level of attention and relaxation during the process of shooting while those who were mid-level archers only increase in attention. Increase in attention and concurrent relaxation allows archers to concentrate on the aiming of the arrow and release it without hesitation or anxiety. Thus, the most ideal psychological state proposed includes both increase in attention and relaxation [2]. However, even elite archers have fluctuation in the psychological condition which may affect their performance. An unstable psychological condition can often induce tension which leads to body stiffness and hamper the archers' performance [1]. However, the belief towards the psychological influence has not been supported with scientific evidence.

Recent studies have shown that the control of autonomic nervous system is important in influencing the performance of archers. When compared with novice players, experienced archers demonstrate an increase in the parasympathetic nervous system activity at the pre-competition level [3]. That leads Carrillo and colleagues to propose that increased parasympathetic tone might provide an optimal performance during a competition. Furthermore, heart rate variability (HRV) was found to be associated with higher parasympathetic activity. The better balance between the parasympathetic and sympathetic activities as reflected by the HRV is beneficial to the performance in sport [4]. Experienced archers exhibited better scoring while at the same time a slower heart rate when compared to inexperienced archers. It is possible that the experience of the archers can help improve the arousal control and, consequently, have better balance between the sympathetic and parasympathetic nervous systems [5]. Therefore, if there is any intervention that can control the arousal control, archers may achieve better performance.

Warm-up exercises such as stretching have been widely adopted to prevent muscular injuries before physically demanding sports [6]. However, this mainly facilitates the physical condition of athletes before engaging sports. On the other hand, there is still no warm-up exercise for archers, which addresses the psychological state in the literature.

Tai Chi, a mind-body exercise, has been shown to promote various health benefits in physical and psychological aspects [7]. Specifically, it has been found to enhance the vagal modulation and decreasing sympathetic activity of the autonomic nervous system [8]. Furthermore, Tai Chi practice has been shown to induce the secretion of serotonin and dopamine which can enhance aminergic synaptic transmission in the central nervous system [9]. These, in turn, help to 
normalize the heart rate and decrease sympathetic activity. The meditation component of Tai Chi has been found to be similar to yoga, which helps to modify age associated decline in HRV in adults as well as enhancing parasympathetic control of the heart [10]. In terms of psychological benefits, Tai Chi has been shown to increase attentiveness and relaxation of novice and skilled practitioners that was reflected by high EEG alpha and beta recordings [11]. Decreased stress and anxiety were also noted in studies with increased EEG theta recordings [12].

It is common that a single arrow shoot-off for score occurs when there are situations, like ties regarding the entrance to the elimination rounds; the progress from one stage of the competition to the next, or for deciding the medal placements after a finals round. Furthermore, if the scores are still the same, the individual whose arrow is closer to the center will win. If the distances are still the same, successive single arrow shoot-offs are required until the tie is resolved. Under such stressful circumstance, archers have reported nervousness, anxiety, tension and tachycardia, all symptoms which could affect their performance.

As mentioned previously, the effects of Tai Chi on the parasympathetic nervous system and mental status could also extend to archery. However, both immediate and long-term effects of Tai Chi practice on archers are unknown. In order to address this, a single-dose of Tai Chi practice may serve as a convenient warm up exercise of archers which may increase the parasympathetic activity of archers, help them to relax and maintain focus that eventually improves their shooting performance. Also, a real-time measurement protocol is much needed during the competition in order to scientifically investigate the effects of intervention on the autonomic nervous activity, brain activity and shooting performance in archers. Therefore, in this pilot study, we aimed at investigating the immediate effect of a session of Tai Chi practice on archers under a simulated stressful situation, and also the design of a feasible measurement protocol during competition. It was hypothesized that the archers in the Tai Chi group would have higher parasympathetic nervous activity, attention and relaxation and shooting scores than the control group who received conventional stretching exercise.

\section{Methods}

\subsection{Participants}

Twenty participants were recruited while one dropped out in the recruitment period due to unavailability. Six males and thirteen female, experienced archers volunteered in a randomized crossover study (Table 1). All participants had no prior experience in practicing Tai Chi. Volunteers were healthy without any cardiovascular, pulmonary or metabolic diseases and were capable of daily activities without limitations. They were not taking hypertensive, anti-depressive or medications that might affect their heart rate. The participants were asked not to consume any alcoholic beverages or caffeinated products, do vigorous exercise for 24 hours, or eat heavy meals before the test. 
Table 1. Demographic characteristics of the participants.

\begin{tabular}{cc}
\hline & Archers $(\mathrm{n}=19)$ \\
\hline Age (years) & $26.0 \pm 7.6$ \\
Archery experience (years) & $7.4 \pm 4.6$ \\
Gender (female: male) & $13: 6$ \\
\hline
\end{tabular}

The study was approved by the Human Subjects Ethics Sub-committee of The Hong Kong Polytechnic University. The procedures were fully explained to all participants and all gave their written informed consent. They were asked to provide their ages, type of bows, archery experience, past medical history and any current medication.

\subsection{Materials}

Two 10-minute videos were taped for each exercise group. The Tai Chi video showed two repetitions of 7 forms of Tai Chi, which included upper limbs, trunk mobilization and rhythmic breathing exercise (Table 2). 10-minute videos were taped because of the practicability in incorporating it into the routine practice of archers. Currently, there is no research on which forms of Tai Chi can enhance archer's performance. The Tai Chi forms were specifically adopted to focus on the upper limbs and trunk movement; mobilize joints of neck, shoulder and trunk; promote eye-hand coordination, and relaxation and attention. For the control group, the stretching video included general stretching exercise of upper limbs and trunk: 1) trunk flexion, 2) bilateral trunk rotation, 3) bilateral cross-arm shoulder and triceps stretch, 4) straight hand behind back, 5) bilateral wrist flexor and extensors stretch \& 6) hands behind neck. Each movement maintained $15 \mathrm{sec}$ hold and repeated for 3 times.

\subsection{Study Design and Measurements}

This was a randomized controlled trial with crossover study design (Figure 1). A pre-test and post-test were conducted to evaluate the effects of Tai Chi on the autonomic nervous system activity \& brain activity (i.e. attention and relaxation) and shooting performance when compared with stretching group (control). Each participant attended each exercise group randomly with at least 1 week of washout period.

The study was held in an indoor public sports center, Cheung Sa Wan Sports Centre where archery practice takes place. All the participants underwent the intervention and testing from $7 \mathrm{pm}-9 \mathrm{pm}$ to ensure a similar experimental environment.

Measurements were conducted in the archery competition site. HRV data were collected using heart rate monitors (RS800CX, Polar Electro Oy, Kempele, Finland) which have been validated for assessment of heart rate variability [13]. For the heart rate variability frequency domain, the ratio (low frequency/high frequency) was reported [14] to demonstrate the balance between sympathetic 


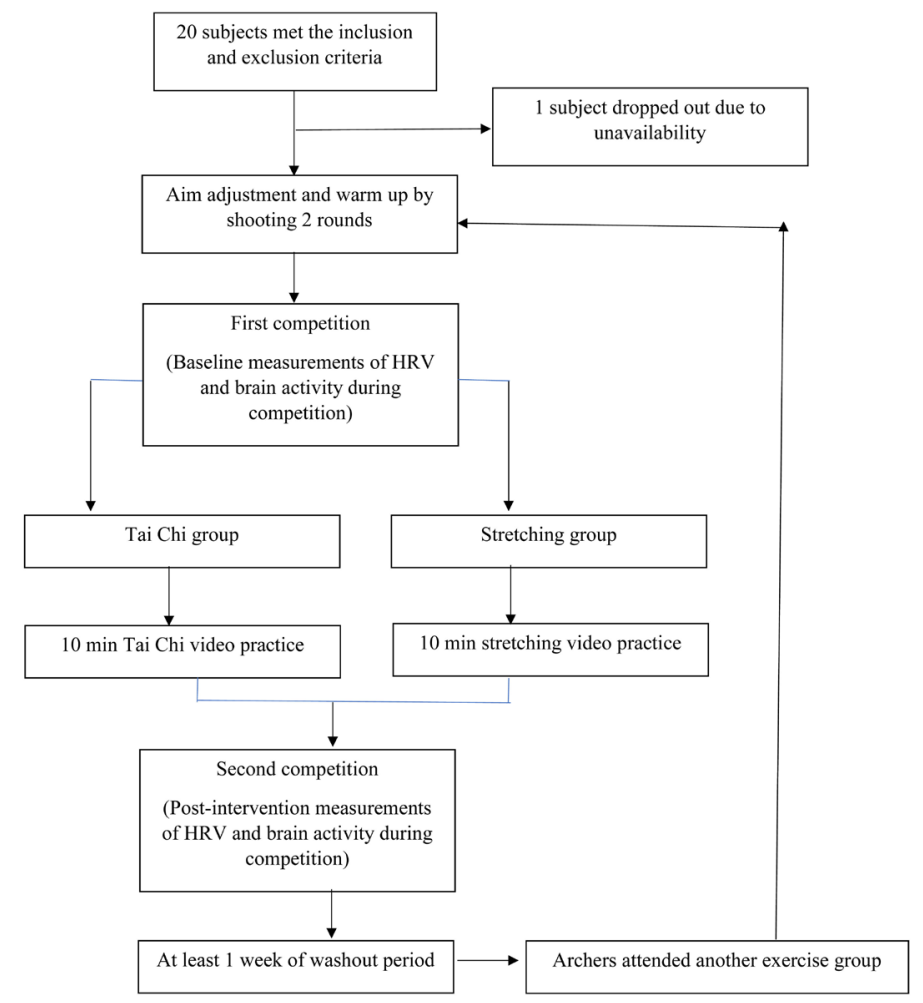

Figure 1. Flow diagram of subjects being investigated in the study.

Table 2. Features and physical functions of the 7 forms of Tai Chi.

\begin{tabular}{|c|c|c|c|}
\hline Form No. & Name & Features & Physical Functions \\
\hline 1 & $\begin{array}{l}\text { Starting } \\
\text { 起式 }\end{array}$ & - Raise hands & $\begin{array}{l}\text { 1) Prepare and relax whole body } \\
\text { 2) Mind concentration } \\
\text { 3) Correct posture }\end{array}$ \\
\hline 2 & $\begin{array}{l}\text { Open and close } \\
\text { 開合 }\end{array}$ & $\begin{array}{l}\text { - Bilateral hands horizontal } \\
\text { abduction and adduction }\end{array}$ & $\begin{array}{l}\text { 1) Relax whole body } \\
\text { 2) Mind concentration } \\
\text { 3) Promote harmonious breathing } \\
\text { 4) Upper limb muscles warm up and joints mobilization }\end{array}$ \\
\hline 3 & $\begin{array}{l}\text { Repulse } \\
\text { monkey } \\
\text { 倒卷肱 }\end{array}$ & $\begin{array}{ll}- & \text { Trunk rotation } \\
- & \text { Weight shifted backwards } \\
- & \text { Lift upper limb } \\
- & \text { Eyes follow the hands movement }\end{array}$ & $\begin{array}{l}\text { 1) Upper limbs and trunk mobilization and muscles activation } \\
\text { 2) Eye-hand coordination } \\
\text { 3) Promote motor and breathing coordination }\end{array}$ \\
\hline 4 & $\begin{array}{l}\text { Work at } \\
\text { shuttles on } \\
\text { both sides } \\
\text { 左右穿梭 }\end{array}$ & $\begin{array}{l}\text { - } \quad \text { Weight shift left and right } \\
-\quad \text { Trunk rotation } \\
-\quad \text { Lift upper limb } \\
-\quad \text { Eyes follow the hands movement }\end{array}$ & $\begin{array}{l}\text { 1) Upper limbs and trunk mobilization and muscles activation } \\
\text { 2) Eye-hand coordination }\end{array}$ \\
\hline 5 & $\begin{array}{l}\text { Wave hands as } \\
\text { clouds } \\
\text { 雲手 }\end{array}$ & $\begin{array}{l}-\quad \text { Weight shift left and right } \\
-\quad \text { Trunk rotation } \\
-\quad \text { Eyes follow the hands movement }\end{array}$ & $\begin{array}{l}\text { 1) Upper limbs and trunk mobilization and muscles activation } \\
\text { 2) Eye-hand coordination }\end{array}$ \\
\hline 6 & $\begin{array}{l}\text { Open and close } \\
\text { 開合 }\end{array}$ & $\begin{array}{l}\text { - Bilateral hands horizontal } \\
\text { abduction and adduction }\end{array}$ & $\begin{array}{l}\text { 1) Relax whole body } \\
\text { 2) Mind concentration } \\
\text { 3) Promote harmonious breathing } \\
\text { 4) Upper limb muscles warm up and joints mobilization }\end{array}$ \\
\hline 7 & Close收式 & - lower hands & 1) Relax whole body and complete the exercise \\
\hline
\end{tabular}


and parasympathetic activity. HRV were analyzed using Nervrokard, Advanced HRV Analysis Software v.13.2.2 (Nevrokard Kiauta, d.o.o., Izola, Slovenia).

Psychological status was monitored by brainwave activities. In general, alpha waves which are from $8-12 \mathrm{~Hz}$ indicate relaxation and meditation while beta waves which are from $13-30 \mathrm{~Hz}$ indicate concentration and attention. Brain wave activities in form of EEG were measured by the MindWave ${ }^{\bullet}$ Neurosky headset (NeuroSky, Inc., CA, USA). It is wireless system commonly used to measure attention and relaxation [15]. Readings of attention and relaxation of archers during the real-time recording periods were generated and retrieved by the Neuroview (NeuroSky, Inc., CA, USA). Both values range from 0 to 100 , with 0 denotes no activity whereas 100 denotes extreme activity in the respective brainwave activities. The algorithm of attention indicates the intensity of mental "focus" or "attention" while the algorithm of meditation shows the level of mental "calmness" or "relaxation". Under normal conditions, the attention level increases when a user focuses on a single thought or an external object and decreases when distracted, while meditation increases when users relax the mind and decreases when they are uneasy or under stress.

Participants had to answer a questionnaire whether their archery shooting was affected by the experimental setup and measurement.

\subsection{Intervention Protocols}

To simulate a competition setting, participants were arranged in pairs (determined by coach based on archers' performance) and competed against each other. They prepared their own archery kits and wore the HRV and EEG devices. They were allowed to adjust the aim of their bows and warm up by shooting 2 rounds. After warm up, they began the first archery competition for the 18-m target. Only one shot was allowed which was considered to add stress among archers. They stayed on the shooting line and waited for the whistle blast for shooting. After warm up, the participants had 2 minutes of standby period and 15 seconds of shooting period. Participants' HRV and brain activity were measured for this period until archers released the arrow. The distance of arrow from the center was measured. They got rest for $10 \mathrm{~min}$ in the audience seats after shooting. This completed the first competition and data were collected for baseline measurements. The procedures of this study are summarized in Figure 1.

Each participant was then randomly assigned to either Tai Chi or stretching group by tossing a coin. They practiced the corresponding exercise according to the 10-minute video with the guidance of an assistant. Once both participants completed their exercises, they were asked to begin the second archery competition and the competition would commence within 5 min. Similarly, HRV, brain activity and shooting performance of archers were recorded with the same duration as in the first competition. Once all archers completed their first assessment, a second assessment was done with treatment groups switched after a wash out period of at least one week. 


\subsection{Statistical Analysis}

Heart rates and EEG of participants throughout the duration of both competitions were recorded and visually inspected prior to analysis. Any abnormal signals e.g. from skin movement artifact were removed and interpolated. The heart rates were computed to generate the low frequency/high frequency ratio while the means of attention and meditation levels were calculated.

All statistical analyses were completed using SPSS 22 software (SPSS Inc., Chicago, IL, USA) with statistical significance set at $\mathrm{p}=0.05$. All data are presented as mean \pm SD. Four variables were evaluated in this study: 1) low frequency to high frequency (LF/HF) ratio, 2) attention, 3) meditation and 4) distance of the arrow from centre $(\mathrm{cm})$. Two-way repeated measure analysis of variance (ANOVA) was performed to compare the treatment and time. Post hoc analysis was performed using paired-t tests for within group comparison. Wilcoxon Signed Ranks Tests were performed for those variables that did not meet the criterion of normality.

\section{Results}

Participants reported that their archery shooting was not affected with the heart rate variability and brain wave measurements.

\subsection{Heart Rate Variability}

Repeated measure ANOVA revealed no significant interaction (Treatment $\times$ Time) in LF/HF ratio.

\subsection{Brainwave Activity}

Repeated measure ANOVA revealed no significant interaction (Treatment $x$ Time) in attention and meditation.

\subsection{Shooting Performance}

Repeated measure ANOVA revealed no significant interaction (Treatment $x$ Time) in the distance of arrow from target center. Nevertheless, further paired $\mathrm{t}$-tests (data normality was met) indicated a statistically significant difference ( $\mathrm{p}$ $=0.021)$ between the before and after treatment measurements in the Tai Chi group. However, the mean distance of Tai Chi group before intervention was $5.58 \mathrm{~cm}(\mathrm{SD}=3.37)$ which was higher than $4.01 \mathrm{~cm}(\mathrm{SD}=3.27)$ in stretching group, although there was no significant difference found in the baseline (Table 3).

\section{Discussion}

The goal of this study was to examine the effects of single-dose of Tai Chi versus stretching interventions on archery performance, attention and meditation, and heart rate variability in experienced archers during a simulated competition. In this crossover study design, we did not find any significant difference among the 
Table 3. Statistical analysis of the outcome measures.

\begin{tabular}{ccccccc}
\hline \multirow{2}{*}{$\begin{array}{c}\text { Outcome } \\
\text { measures }\end{array}$} & \multicolumn{2}{c}{ Stretching } & \multicolumn{2}{c}{ Tai Chi } & \multicolumn{2}{c}{ p value } \\
\cline { 2 - 7 } & Before & After & Before & After & $\begin{array}{c}\text { Treatment } \\
\text { (main effect) }\end{array}$ & $\begin{array}{c}\text { Time } \\
\text { (main effect) }\end{array}$ \\
\hline LF/HF ratio & $1.10 \pm 0.41$ & $1.18 \pm 0.62$ & $1.24 \pm 0.41$ & $1.03 \pm 0.41$ & 0.959 & 0.184 \\
Attention (\%) & $47.15 \pm 8.69$ & $46.67 \pm 8.51$ & $48.03 \pm 10.24$ & $48.49 \pm 7.55$ & 0.708 & 0.892 \\
Meditation (\%) & $53.92 \pm 7.40$ & $53.34 \pm 8.75$ & $54.80 \pm 7.81$ & $51.66 \pm 9.03$ & 0.777 & 0.329 \\
Distance (cm) & $4.01 \pm 3.27$ & $4.49 \pm 3.69$ & $5.58 \pm 3.37$ & $4.49 \pm 2.73$ & 0.386 & $0.030^{*}$ \\
\hline
\end{tabular}

${ }^{*}$ Denotes a difference at the significance level of $\mathrm{p}<0.05$.

participants in the aforementioned measures under the two intervention conditions. However, the subjects after practicing Tai Chi was found to improve in their shooting performance. The improvement cannot be explained by the outcomes of HRV and brain wave activities as there was no within group difference. On the other hand, Tai Chi practice may affect the other factors such as fitness and skills that might improve the shooting performance e.g. eye-hand coordination [16]. However, this was not measured in this pilot study. Other factors like enhanced motor control and stabilization of upper limb, shoulder and trunk are also possible mechanisms to explain the better performance. Further study related to motor control is warranted.

In this pilot study, the Tai Chi intervention did not alter the parasympathetic nervous system and attention and relaxation activities as differed from previous investigation. One possible explanation is because each participant needed to learn a new exercise, Tai Chi on the spot, just before the measurement. Although Tai Chi could improve attention and meditation of skilled or novice practitioners ( $<1$ year of experience) in middle-aged women [11], learning a new exercise just before archery competition might exert stress on participants that hinder the attention and relaxation effects of Tai Chi. Under stressful circumstance, two systems will be activated in respond to stress: 1) sympathetic nervous system and 2) the hypothalamic-pituitary-adrenal (HPA) axis. Sympathetic nervous system will lead to increase of catecholamine adrenaline and noradrenaline from the adrenal medulla, which will cause increase in heart rate and release of glucocorticoids that leads to a state of arousal [17]. On the other hand, the HPA axis is a collective group of structures localized in the hypothalamus, anterior lobe of pituitary gland, and adrenal gland. In response to stress, corticotrophin-releasing factor are secreted for the release of glucocorticoids from the adrenal cortex [18]. Together, these two systems are suggested to override the effects of Tai Chi on parasympathetic activity, attention and relaxation. This learning stress suggests why the performance of archers in Tai Chi group did not achieve favourable findings than those after stretching intervention.

Secondly, most of our participants were experienced archers, thus the effects of Tai Chi might be minimal as ceiling effect might have occurred. The amounts 
of stress perceived by the experienced archers are usually less than those novice archers. The effect of single-dose protocol of Tai Chi might be too minimal to have a detectable impact on their parasympathetic nervous system or mental status. Research from Carrillo et al. (2011) [3] supported the idea that experienced archers already have more relaxed status than the novice. They found that experienced archers showed an increase in parasympathetic system activity during competition while novice archers remain unaltered. With a highly activated parasympathetic nervous system in experienced archers, further activation of the system by short-term Tai Chi practice may be minimal.

Thirdly, the time practicing Tai Chi was relatively short and also the effects of Tai Chi might not be shown immediately after practice. Since the aim of this study was to examine the effect of Tai Chi as a warm up exercise, the practicing was only ten minutes. To our knowledge, there was no current literature investigating the immediate effect of a single session of Tai Chi practice (10 min) on new learners. Two current systematic reviews studied the effects of different modes of warm up on exercise performance haven't found studies employing Tai Chi as a warm up exercise [19] [20]. The effective length of Tai Chi exercise and duration to reach peak effect remains unknown. Current literature only studied the effect of post Tai Chi exercise (practise for 40 - $60 \mathrm{~min}$ ) on those experienced Tai Chi practitioners (>1 year) [7]. Lu \& Kuo (2003) [21] reported that a 40-min Tai Chi practice increased the parasympathetic activity and decreases sympathetic activity of older Tai Chi practitioners after $30 \mathrm{~min}$ and $60 \mathrm{~min}$ post Tai Chi exercise. Recent research from Kalsaria and colleagues (2012) [22] further confirmed and extended the study of Lu \& Kuo. Kalsaria and colleagues found the same pattern of cardiac autonomic modulation of Tai Chi exercise ( 1 hour) after $30 \mathrm{~min}$ post practice but not $10 \mathrm{~min}$ or $20 \mathrm{~min}$. In contrast with our Tai Chi exercise protocol, our Tai Chi practice was short and the effect of Tai Chi might not be immediately shown right after practising Tai Chi. The effective length of Tai Chi practice and duration to reach peak effect after exercise still required further study.

A major limiting factor in this pilot study is that the sample size was small. More participants are needed to be recruited in the future main study. In consideration of future protocol, we suggest that for future randomized controlled trial, participants should have more practice sessions of Tai Chi before measurement which might reduce the learning stress. Two 10-min Tai Chi warm up sessions weekly for 12 weeks can be implemented before the start of archery practice. Furthermore, longer practising time of warm up might be required to induce a more significant effect of Tai Chi according to the previous study by $\mathrm{Lu}$ \& Kuo (2003) [21]. Moreover, the archers will practise Tai Chi at least $30 \mathrm{~min}$ earlier to the competitions to optimize the relaxation and meditation effect of Tai Chi. For additional research, novice archers may be a target group to verify whether the effect of Tai Chi is more significant in this group as they may be more prone to the relaxation and meditation effect of Tai Chi. 


\section{Conclusion}

In conclusion, there was no significant difference of autonomic nervous system activity, attention, relaxation and shooting performance between Tai Chi and stretching interventions. There was a significant improvement observed in the performance of archers after Tai Chi intervention when compared with baseline. Moreover, this pilot study showed the feasibility of performing a real-time measurement in the competition field and it did not hinder the archer's performance. Therefore, future study may consider such measurement protocol to examine the possible benefits of Tai Chi practice on archers.

\section{Conflicts of Interest}

The authors declare no conflicts of interest regarding the publication of this paper.

\section{References}

[1] Kim, H.B., Kim, S.H. and So, W.Y. (2015) The Relative Importance of Performance Factors in Korean Archery. The Journal of Strength \& Conditioning Research, 29, 1211-1219. https://doi.org/10.1519/JSC.0000000000000687

[2] Lee, K. (2009) Evaluation of Attention and Relaxation Levels of Archers in Shooting Process Using Brain Wave Signal Analysis Algorithms. Emitional Science, 12, 341-350.

[3] Carrillo, A.E., Christodoulou, V.X., Koutedakis, Y. and Flouris, A.D. (2011) Autonomic Nervous System Modulation during an Archery Competition in Novice and Experienced Adolescent Archers. Journal of Sports Sciences, 29, 913-917. https://doi.org/10.1080/02640414.2011.568514

[4] Lo, C.T., Huang, S.H. and Hung, T.-M. (2008) A Study of the Relationship between Heart Rate Variability and Archery Performance. International Journal of Psychophysiology, 69, 276-316. https://doi.org/10.1016/j.ijpsycho.2008.05.231

[5] Clemente, F., Couceiro, M., Rocha, R. and Mendes, R. (2011) Study of the Heart Rate and Accuracy Performance of Archers. Journal of Physical Education \& Sport, 11, 434-437.

[6] Woods, K., Bishop, P. and Jones, E. (2007) Warm-Up and Stretching in the Prevention of Muscular Injury. Sports Medicine, 37, 1089-1099.

https://doi.org/10.2165/00007256-200737120-00006

[7] Field, T. (2011) Tai Chi Research Review. Complementary Therapies in Clinical Practice, 17, 141-146. https://doi.org/10.1016/j.ctcp.2010.10.002

[8] Figueroa, M.A., DeMeersman, R.E. and Manning, J. (2012) The Autonomic and Rate Pressure Product Responses of Tai Chi Practitioners. North American Journal of Medical Sciences, 4, 270-275. https://doi.org/10.4103/1947-2714.97208

[9] Zhang, L., Layne, C., Lowder, T. and Liu, J. (2012) A Review Focused on the Psychological Effectiveness of Tai Chi on Different Populations. Evidence-Based Complementary and Alternative Medicine, 2012, Article ID: 678107.

[10] Yunati, M.S., Deshpande, V. and Yuwanate, A.H. (2014) Dynamics of Heart Rate Induced by Sahaja Yoga Meditation in Healthy Normal Subjects above 40 Years. National Journal of Physiology, Pharmacy and Pharmacology, 4, 80-85. https://doi.org/10.5455/njppp.2014.4.100920131 
[11] Liu, Y., Mimura, K., Wang, L. and Ikuda, K. (2003) Physiological Benefits of 24-Style Taijiquan Exercise in Middle-Aged Women. Journal of Physiological Anthropology and Applied Human Science, 22, 219-225. https://doi.org/10.2114/jpa.22.219

[12] Field, T., Diego, M. and Hernandez-Reif, M. (2010) Tai Chi/ Yoga Effects on Anxiety, Heartrate, EEG and Math Computations. Complementary Therapies in Clinical Practice, 16, 235-238. https://doi.org/10.1016/j.ctcp.2010.05.014

[13] Gamelin, F.X., Berthoin, S. and Bosquet, L. (2006) Validity of the Polar S810 Heart Rate Monitor to Measure R-R Intervals at Rest. Medicine and Science in Sports and Exercise, 38, 887-893. https://doi.org/10.1249/01.mss.0000218135.79476.9c

[14] Task Force of the European Society of Cardiology and the North American Society of Pacing and Electrophysiology (1996) Heart Rate Variability: Standards of Measurement, Physiological Interpretation and Clinical Use. Circulation, 93, 1043-1065. https://doi.org/10.1161/01.CIR.93.5.1043

[15] Crowley, K., Sliney, A., Pitt, I. and Murphy, D. (2010) Evaluating a Brain-Computer Interface to Categorise Human Emotional Response. 2010 10th IEEE International Conference on Advanced Learning Technologies, Sousse, 5-7 July 2010, 276-278. https://doi.org/10.1109/ICALT.2010.81

[16] Pei, Y.C., Chou, S.W., Lin, P.S., Lin, Y.C., Hsu, T.H. and Wong, A.M. (2008) Eye-Hand Coordination of Elderly People Who Practice Tai Chi Chuan. Journal of the Formosan Medical Association, 107, 103-110. https://doi.org/10.1016/S0929-6646(08)60123-0

[17] Schwabe, L., Joëls, M., Roozendaal, B., Wolf, O.T. and Oitzl, M.S. (2012) Stress Effects on Memory: An Update and Integration. Neuroscience \& Biobehavioral Reviews, 36, 1740-1749. https://doi.org/10.1016/j.neubiorev.2011.07.002

[18] Smith, S.M. and Vale, W.W. (2006) The Role of the Hypothalamic-Pituitary-Adrenal Axis in Neuroendocrine Responses to Stress. Dialogues in Clinical Neuroscience, 8, 383.

[19] Fradkin, A.J., Zazryn, T.R. and Smoliga, J.M. (2010) Effects of Warming-Up on Physical Performance: A Systematic Review with Meta-Analysis. Journal of Strength and Conditioning Research, 24, 140-148. https://doi.org/10.1519/JSC.0b013e3181c643a0

[20] McCrary, J.M., Ackermann, B.J. and Halaki, M. (2015) A Systematic Review of the Effects of Upper Body Warm-Up on Performance and Injury. British Journal of Sports Medicine, 49, 935-942. https://doi.org/10.1136/bjsports-2014-094228

[21] Lu, W.A. and Kuo, C.D. (2003) The Effect of Tai Chi Chuan on the Autonomic Nervous Modulation in Older Persons. Medicine and Science in Sports and Exercise, 35, 1972-1976. https://doi.org/10.1249/01.MSS.0000099242.10669.F7

[22] Kalsaria, P., Li, H., Waite, G., Moga, M., Kingsley, J.D. and Geib, R.W. (2012) Acute Effects of Tai Chi Exercise on Cardiac Autonomic Function in Healthy Adults with Tai Chi Experience. Biomedical Sciences Instrumentation, 48, 226-231. 